\title{
Alexandre Mallard, Petit dans le marché. Une sociologie de la très petite entreprise
}

Paris, Transvalor-Presses des Mines, coll. « Sciences sociales », 2011

\section{Alina Surubaru}

\section{OpenEdition}

\section{Journals}

Édition électronique

URL : https://journals.openedition.org/travailemploi/6170

DOI : 10.4000/travailemploi.6170

ISSN : 1775-416X

Éditeur

DARES - Ministère du Travail

Édition imprimée

Date de publication : 14 décembre 2013

Pagination : 88-89

ISSN : 0224-4365

\section{Référence électronique}

Alina Surubaru, «Alexandre Mallard, Petit dans le marché. Une sociologie de la très petite entreprise », Travail et Emploi [En ligne], 136 | octobre-décembre 2013, mis en ligne le 01 octobre 2013, consulté le

31 juillet 2021. URL : http://journals.openedition.org/travailemploi/6170 ; DOI : https://doi.org/

10.4000/travailemploi.6170

Ce document a été généré automatiquement le 31 juillet 2021.

(C) Direction de l'animation de la recherche, des études et des statistiques (Dares) 


\section{Alexandre Mallard, Petit dans le marché. Une sociologie de la très petite entreprise}

Paris, Transvalor-Presses des Mines, coll. « Sciences sociales », 2011

\section{Alina Surubaru}

\section{RÉFÉRENCE}

Alexandre Mallard, Petit dans le marché. Une sociologie de la très petite entreprise, Paris, Transvalor-Presses des Mines, coll. « Sciences sociales », 2011, 264 p.

1 Cet ouvrage propose une sociologie de la très petite entreprise (TPE), en s'appuyant à la fois sur des données quantitatives et qualitatives (enquête TELUS). Les données quantitatives ont été recueillies en 2000 dans le cadre des travaux menés pour le compte des unités d'affaires chargées du marché des professionnels à France Télécom. Après trois mois d'observation du trafic téléphonique de 881 TPE, deux enquêtes par téléphone ont été réalisées auprès d'environ 800 de ces entreprises. Ce travail a été mené par Alexandre Mallard et Laurence Dhaleine, l'enquête par sondage étant effectuée à l'Institut TNS Sofres. Ensuite, dans le cadre d'un contrat de recherche passé entre France Télécom et l'École des mines de Nantes, Sophie Dubuisson-Quellier a réalisé 36 entretiens avec des responsables de TPE situées dans la région nantaise, avec pour but de mieux comprendre le rôle du téléphone fixe comme médiation marchande.

2 Si ces différentes données ont déjà donné lieu à des publications, l'ambition d'Alexandre Mallard est de revenir sur l'ensemble des variables utilisées dans le cadre de l'enquête TELUS afin de répondre à une question apparemment simple : que signifie " être petit dans le marché ? "Cela va de soi, il n'y a pas une, mais de multiples façons d'être petit dans le marché et sur ce point, l'auteur est particulièrement vigilant. Entre l'opticien ou la mercière-couturière qui attendent les clients dans leurs boutiques et l'électricien ou l'infirmière qui sillonnent la ville pour se rendre chez eux, les 
expériences marchandes ne sont pas équivalentes et les appariements entre offre et demande, bien divers. Or, en relevant le pari risqué des comparaisons, l'auteur tente justement d'enrichir la compréhension sociologique des transactions commerciales de proximité, dans une perspective théorique inspirée par les travaux de Michel Callon ${ }^{1}$ et Bruno Latour².

3 Les trois premiers chapitres de l'ouvrage décrivent la formation de l'expérience marchande de ces acteurs, puis montrent ce qui permet aux TPE de s'ajuster de manière flexible à leur environnement. Ensuite, les deux derniers chapitres explorent le rôle d'un dispositif technique particulier, le téléphone fixe, et pointent l'importance de l'interface communicationnelle, c'est-à-dire de l'ensemble des situations de communications qui mettent en interaction une entreprise avec sa clientèle et ses partenaires.

4 Conçue comme " extrêmement socialisée, au sens où la relation sociale y joue un rôle central ", l'expérience marchande est ici "équipée, au sens où de multiples informations ne pourraient émerger, être mises en perspective, stockées et remobilisées sans le concours de dispositifs techniques variés» (p. 76). Cinq processus apparaissent ensuite comme déterminants pour la formation de l'expérience marchande: l'expérimentation (le tâtonnement autour du bien économique et de la situation d'échange), la comptabilisation (l'exploitation des données à caractère comptable), l'imprégnation de l'environnement local et global (l'acquisition et l'appropriation des informations plus ou moins générales), l'interaction (les pratiques de sociabilité avec les clients) et la triangulation (la perception de l'environnement au travers d'une focale multiple, ajustant des informations issues du marché tel que le professionnel le voit et des informations qualifiant le marché tel qu'il est vu par les autres).

5 Loin d'être simple ou linéaire, l'expérience marchande des TPE apparaît donc comme un processus à multiples facettes. Le mérite de l'auteur, et plus généralement de l'enquête TELUS, est de dépasser l'idée intuitive selon laquelle les organisations peu complexes (c'est-à-dire au sein desquelles la division du travail n'est pas toujours instituée) obéiraient à des logiques économiques ou sociales prédéfinies. Or, pour pouvoir tenir la promesse d'une telle perspective, Alexandre Mallard adopte dans son ouvrage une analyse en termes de "capacités commerciales", qu'il emprunte à la tradition évolutionniste en économie. Ainsi, il s'efforce de montrer comment les compétences individuelles et collectives, les routines et les savoir-faire commerciaux se conjuguent singulièrement pour rendre possible l'insertion des TPE dans un environnement économique donné. Ce faisant, il reprend à son compte l'idée selon laquelle l'ajustement marchand porte de façon centrale non seulement sur ce qui est échangé, mais aussi sur les préférences des partenaires de l'échange ${ }^{3}$. En d'autres termes, lorsqu'un client s'adresse à une couturière ou à un maçon, il a déjà une idée plus ou moins claire du type de service attendu. Pourtant, les modalités concrètes de déroulement des transactions dépendent moins de ces facteurs que du jeu d'acteurs et des négociations à l'œuvre : "Concernant le prix des retouches, il y a un forfait de base, puis après c'est à moi de juger, de voir avec la cliente", affirme par exemple $\mathrm{M}^{\text {me }}$ Lemerle, mercière et couturière dans les faubourgs de Nantes (p. 79). «Le client commence par nous montrer ce qu'il veut et ensuite il y a une étude de réalisation. Parce qu'il y a des fois où les clients arrivent avec des jolis plans faits par des architectes, ils sont magnifiques, les maisons 
sont très, très belles mais elles ne sont pas réalisables ", précise un responsable de travaux en bâtiment (p. 106).

$6 \quad$ Au-delà d'une simple description des négociations marchandes, Petit dans le marché vise surtout à rendre intelligible les différentes ressources qui permettent aux TPE de s'assurer une place dans l'environnement économique. Cinq capacités sont ainsi identifiées et minutieusement analysées : la capacité à capter la clientèle, la capacité à coproduire des appariements singuliers entre l'offre et la demande, la capacité à articuler le lien marchand et le lien social, la capacité à couvrir l'espace et la capacité à synchroniser le marché et la production. Ces différentes capacités expliquent sans surprise le rapport privilégié que semblent entretenir les TPE avec le territoire, mais aussi les conséquences sur les rythmes de vie de leurs responsables (équilibre difficile entre la vie privée et la vie professionnelle). Étant donné ce cadre conceptuel, l'enquête sur les usages du téléphone fixe trouve entièrement sa place. Cet objet presque désuet de nos jours rend bien compte de la pluralité des schémas d'activité et des formes d'organisation possibles autour d'un même dispositif : par exemple, collecter et mettre en forme la demande économique, organiser la rencontre entre le professionnel et le client dans l'espace-temps, entretenir la sociabilité.

$7 \mathrm{Au}$ terme de cette lecture, deux remarques générales s'imposent. Solidement documentée et argumentée, la posture théorique d'Alexandre Mallard aboutit néanmoins à un paradoxe. Si on suit volontiers l'auteur dans son pari de définir ce que signifie être petit dans le marché, il n'en reste pas moins que ses conclusions ne sont pas faciles à appréhender, tant la richesse des situations économiques est grande. Entre la toiletteuse de chiens et l'éleveur de gibier de chasse, le libraire et le négociant mandataire, l'infirmière et l'agent immobilier, le lecteur risque parfois de se perdre. Si l'auteur veille à ce que l'on s'y retrouve toujours - et l'on rend ici hommage à la grande rigueur intellectuelle dont il fait preuve -, un autre problème est cependant à souligner. La montée en généralité qu'il opère se fait parfois au prix d'une simplification de l'histoire des transactions marchandes évoquées, simplification qui a pour principal effet de jeter un voile sur les éléments constitutifs des différentes capacités commerciales décrites. Au final, le lecteur risque donc de rester un peu sur sa faim, car il ne saura jamais vraiment pourquoi la couturière n'a pas voulu faire un prix à son client : est-ce sa tête qui ne lui revient pas ou bien n'en fait-elle qu'à sa tête ?

\section{NOTES}

1. Callon M. (1998), The laws of the markets, Oxford, Blackwell.

2. Latour B. (1994), «Une sociologie sans objet? Remarques sur l'interobjectivité », Sociologie du travail, vol. 36, $\mathrm{n}^{\circ} 4$, pp. 587-607.

3. Callon M., Meadel C., Rabeharisoa V. (2000), «L'économie des qualités », Politix, vol. 13, n52, pp. 211-239. 


\section{AUTEURS}

\section{ALINA SURUBARU}

École des mines de Nantes 